\title{
Racial, Gender, and Professional Diversification in the Forest Service: A Rejoinder
}

\section{Paul Mohai and Jennifer C. Thomas}

We appreciate Christopher Simon's catching our error. Of course, he is right that we should have divided by the 1983 rather than the 1992 numbers in computing the percentage changes between those two years. Tables 3 and 4 produced by him contain the correct percentages. We regret any confusion that our error caused.

Christopher Simon is correct that the percentage changes we indicated in Tables 1 and 2 are, for the most part, greater than what we had reported. However, this fact does not appear to contradict nor change appreciably our principal findings and conclusions. We pointed out in our paper (Thomas \& Mohai, 1995, p. 302) that: "Figure 2 [which was not affected by our miscalculations] shows that the percentage of women in the agency as a whole increased from $30.0 \%$ in 1983 to $40.3 \%$ in 1992." We also acknowledged (Thomas \& Mohai, 1995, p. 302) that "[w]omen in the Forest Service made gains in nearly every job category" and that "[t]he average grade of women increased significantly during the decade." However, even when the corrected percentages in Tables 1 and 2 are taken into account, this does not change our finding (Thomas $\&$ Mohai, 1995, p. 303) that: "Despite these changes, the percentages of women in each category relative to each other stayed about the same (Figure 3)." Note that this latter finding was based on examination of the relative percentages of women in each of the job categories displayed in Figure 3, which were not affected by the miscalculations in Table 1 and are correct. We went on to say (Thomas \& Mohai, 1995, p. 303):

In other words, Clerical remains the category with the highest percentage of women, Administration is second, followed by Social Science and Recreation. Biological Science and Physical Science switched places but remain the "intermediate" categories, while the percentages of women in Forestry. Range, and Engineering remain lower than other categories. Women thus remain concentrated in the categories that will not lead to line positions.

This finding is an important one and rests entirely on the information provided in Figure 3. It can be deduced, as well, from the absolute numbers in each of the job categories for 1992 that are reported in Table 1.

Similarly, in the case of racial diversification, we pointed out (Thomas \& Mohai, 1995, p. 303) that: "while people of color accounted for just $10.1 \%$ of the agency in 1983, they comprised $14.7 \%$ of all employees in 1992 (Figure 2)." We also acknowledged (Thomas \& Mohai, 1995, p. 303) that: "people of color made gains in nearly all job categories (Table 2 and Figure 4)." As before, these findings and conclusions are unchanged. Also, just as in the case of women, people of color remain concentrated in the Clerical and Administration categories despite the gains made in other job categories; and as before, these findings are unrelated to the miscalculated percentages reported in Table 2. They are based on 
the results displayed in Figure 4, and likewise can be deduced from the absolute numbers of people of color in each of the job categories for 1992 that are reported in Table 2.

We acknowledged in our "Conclusions" that the Forest Service appears to be making gains in the professional, gender, and racial diversification of the agency. We acknowledged (Thomas \& Mohai, 1995, p. 307) that: "The Forest Service's efforts to bring women into the agency appear to have been quite successful. Many more women are employed by the Forest Service now than in 1983 , and the agency is much closer to matching the civilian workforce percentages than before." We also acknowledged (Thomas \& Mohai, 1995, p. 307) that: "Clearly, the total numbers and percentages of people of color with the Forest Service have risen over the past decade." We concluded furthermore (Thomas \& Mohai, 1995, p. 307) that these gains "are encouraging for those who view workforce diversification as an important goal for the agency." At the same time, however, it remains true that, in spite of the gains made by women and people of color, "[w]omen remain significantly underrepresented in the Forestry, Engineering, and Range categories, from which most line officers are chosen" (Thomas \& Mohai, 1995, p. 307) and that they continue to be "overrepresented in the stereotypically 'female' jobs like those in Clerical and Administration" (Thomas \& Mohai, 1995, p. 307). Likewise, in spite of their gains, "[f]or the most part, today people of color seem to be concentrated in the lowest levels of the agency" (Thomas \& Mohai, 1995, p. 307). These conclusions do not change, even with the revised calculations provided by Christopher Simon.

Paul Mohai is associate professor at the School of Natural Resources and Environment at the University of Michigan in Ann Arbor.

Jennifer C. Thomas is a mediator with the Keystone Center, a nonprofit organization specializing in environmental policy dispute resolution. She recently earned her master's degree at the University of Michigan's School of Natural Resources and Environment.

\section{Reference}

Thomas, J. C., \& Mohai, P. (1995). Racial, gender, and professional diversification in the forest service from 1983 to 1992. Policy Studies Journal, 23(2), 296-309. 
This document is a scanned copy of a printed document. No warranty is given about the accuracy of the copy. Users should refer to the original published version of the material. 